\title{
Surgical Treatment of Metastatic Tumors to the Lungs
}

\author{
James B. D. Mark, M. D.
}

It is easy to understand why tumors in various parts of the body metastasize to lungs. The pulmonary capillary bed is the first filter that many circulating tumor cells reach. Which cells actually form pulmonary metastases depends on a number of physical, immunologic and biochemical factors. Over $30 \%$ of systemic tumors have lung metastases by the time the patient dies but only a select few of these patients are candidates of resection of their tumors.

There has never been a prospective, randomized, controlled series reported of treatment of metastatic tumors to the lungs. All reports are retrospective. The earliest reports were from major cancer hospitals and included the institutional experience with treatment of metastatic carcinomas and sarcomas. More recently, site-specific series have been reported.

In almost all cases, the objective of treatment should be the complete removal of all known tumor. Guidelines for patient selec- tion have evolved over the years. Our criteria are as follows:

- On the basis of preoperative evaluation, complete removal of all tumor should be thought possible.

- The planned operation should be accompanied by low risk to the patient.

- The primary tumor should be under control.

- There should be no metastases elsewhere.

- There should be no other potentially curative treatment available.

Using these criteria we have operated on over 200 patients during the past 20 years. Overall, three year survival rate has been in the range of $50 \%$ and five year survival rate $40 \%$. In general, patients with multiple metastases fare as well as those with solitary metastases. Conservative or limited resections are sufficient for treatment of patients with metastatic tumors to the lungs. 